\title{
A New, Rapid and Efficient HPLC Method to Assay Resveratrol in Food Supplements
}

\begin{abstract}
VERONICA ISABELA CRACIUN ${ }^{1 *}$, FELICIA GABRIELA GLIGOR², ANCA MARIA JUNCAN², ADRIANA AURELIA CHIS ${ }^{1,2}$, LUCA LIVIU RUS 1,2

'Polisano Pharmaceuticals SA, 156 Alba Iulia Blvd., 550052, Sibiu, Romania

¿Lucian Blaga University of Sibiu, Faculty of Medicine, Preclinical Department, Pharmacy Specialization, 2A Lucian Blaga Str., 550169 Sibiu, Romania

The aim of this study consisted in the development of a rapid and reliable high performance liquid chromatography (HPLC) method for resveratrol identification and assay in food supplements, trying to offer a robust, direct and convenient solution. By this method, several supplements with varying resveratrol content, between 103 and 119\% of the stated amount per dosage form, were analyzed.
\end{abstract}

Keywords: resveratrol, HPLC method, dietary supplements

Resveratrol has numerous benefits for human health: antioxidant, anti-inflammatory and cytoprotective [1]. Resveratrol is very abundant in beverages such as red wine, fruits such as grape berry and particularly in dried roots of the plant Polygonum cuspidatum. Also, resveratrol can be found in other natural foods such as bilberries, blueberries, peanuts, pistachios and in valuable by-products like grape skin and grape pomace $[2,3]$.

Resveratrol has multiple benefits. Among these are:

-facilitation of increased oxidation of fatty acids, mitochondrial biogenesis, mitochondrial respiration, and gluconeogenesis.

-inhibits expression of HIF- $1 \alpha$ and VEGF, explaining its effective action against cancer.

-triggers apoptosis of activated T cells and suppresses proinflammatory molecules, and thus is of benefit in autoimmune diseases.

-alters gut microbiota and influences stem cell proliferation and differentiation [4].

According to its chemical structure, resveratrol $(3,42,5-$ trihydroxystilbene, Fig.1.) is a polyphenolic compound. It is similar to diethylstilbestrol, a synthetic estrogen $[4,5]$.

Resveratrol has been the subject of intense interest in recent years due to a range of unique anti-aging properties.These include cardiovascular benefits via increased nitric oxide production, down-regulation of vasoactive peptides, lowered levels of oxidized low-density lipoprotein, and cyclooxygenase inhibition; possible benefits on Alzheimer disease by breakdown of betaamyloid and direct effects on neural tissues; phytohormonal actions; anticancer properties [6].<smiles>Oc1ccc(/C=C/c2cc(O)cc(O)c2)cc1</smiles>

Fig. 1. Chemical structure of trans-resveratrol

Antioxidant preservatives are able to inhibit reactions promoted by oxygen, thus avoiding the oxidation and rancidity of commonly used fats, oils, waxes, surfactants, perfumes, etc. $[7,8]$. Because of this, resveratrol can be used on one hand in manufacturing of food supplements and on the other hand, due to its antioxidant effects, in the manufacturing of cosmetic products, industry which increasingly focuses on the development of active cosmetics $[9,10]$.

In order to supply this molecule in high concentrations, the food supplements market offers various products. Also, innovative pharmaceutical forms containing resveratrol are being tested [11]. In most supplements, resveratrol (and other antioxidants) is combined with various plantextracts containing polyphenols in order to enhance healthpromoting effects [12], including antioxidative properties [13]. Also selling supplements can reduce costs and optimize the activity of pharmaceutical units [14]. The presence of the actual content stated by the producers is crucial for the therapeuticbenefits and an indicator of product quality [15].

May review papers were published describing pharmacological properties and potential use of resveratrol in human therapy [16-27]. As a consequence the interest of analysing resveratrol is continuously growing.

Many papers were published describing quantitative determination in different matrices (wines, fruits, honey, plants and plant extracts, grape pomace, pharmaceutical dosage forms, biological fluids, etc): HPLC and UPLC with different detection [28-37], GC [38-40], CE [41-43], spectrofluorimetry [45-46], voltammetry [47, 48], MEKC [49], by amperometric biosensor [50], by electrochemiluminescence [51] and by resonance light scattering technique [52].

Due to their characteristics (selectivity, specificity, accuracy) chromatographic methods were successfully applied for quantitative analysis of: pharmaceuticals [53, $54]$, plants [55] and cosmetics [8, 56, 57].

\section{Experimental part \\ Materials, reagents and standards}

Three food supplements and a standardized extract of Polygonium Cuspidatum were analyzed. Food supplements have been purchased from pharmacies and otherspecialized stores.

The supplements analyzed were coded as follows:

-PB001 - is presented as hard gelatin capsules containing $50 \mathrm{mg}$ resveratrol/cps. 
-PB002 - is presented as hard gelatin capsules containing $50 \mathrm{mg}$ resveratrol/cps.

-PB003 - is presented as hard gelatin capsules containing $100 \mathrm{mg}$ resveratrol/cps, $95 \mathrm{mg}$ proanthocyanidines from grape seeds/cps. and $60 \mathrm{mg}$ coenzyme Q10/cps.

-PB004 - is a Polygonium Cuspidatum extract with a minimum resveratrol content of $50 \%$.

Resveratrol was extracted with analytical purity methanol from VWR.

Resveratrol CRS (99.6\%) from EDQM was used as external standard.

\section{Sample and standard preparation}

From each product 20 capsules were taken and the average mass was performed. The sample solution was prepared using $50 \mathrm{mg}$ of pow der content from 20 capsules in $50 \mathrm{~mL}$ of methanol and sonicated for $15 \mathrm{~min}$. From this solution $1 \mathrm{~mL}$ was diluted ten times using the same solvent.

The standard solution was prepared in the same manner. The standard and sample solutions were previously filtered using $0.45 \mu \mathrm{m}$ filters before injection. The standard was injected six times and each sample was prepared in triplicate and injected once.

\section{Equipment and chromatographic conditions}

The identification and quantification of resveratrol was performed using a HPLC method developedon basis of the European Pharmacopoeia. The separation of resveratrol was performed by HPLC using an Agilent 1200 HPLC system consisting of a quaternary pump, degassing device, autosampler, diode array detector (PDA), and Agilent ChemStation software. A Zorbax SB-C18, 250x4.6 $\mathrm{mm}, 100 \AA$ (S/No86996-11, B/No:5701-029) column was used. The mobile phase used for isocratic elution consisted of distilled water (MilliQ purity, $0.5 \mu \mathrm{S} / \mathrm{cm}$ at $20^{\circ} \mathrm{C}$ )-methanol HPLC grade (60: 40) (v/v). The acquisition parameters used were: flow rate $1.5 \mathrm{~mL} / \mathrm{min}$, injection volume $10 \mu \mathrm{L}$, column temperature $25^{\circ} \mathrm{C}$ and detection was performed at 303 $\mathrm{nm}$.

\section{Results and discussions}

Identification was made by comparison of retention times and by spectral data of PDA with those obtained for the standard as is shown in figure 2 and figure 3 . The retention time of resveratrol was approximatively $8.8 \mathrm{~min}$.
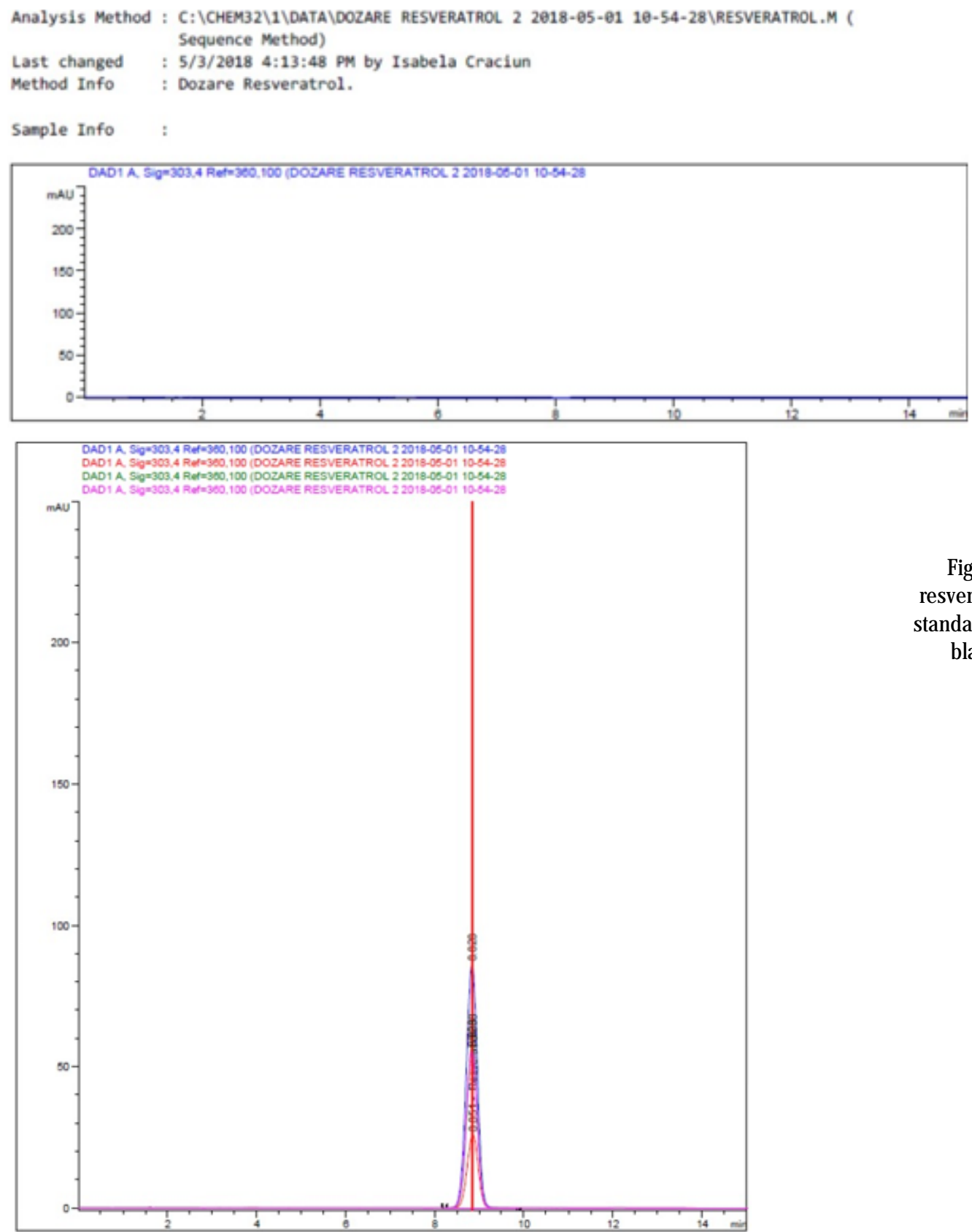

Fig.2. Retention times of resveratrol for samples and standard chromatograms and blank chromatogram 


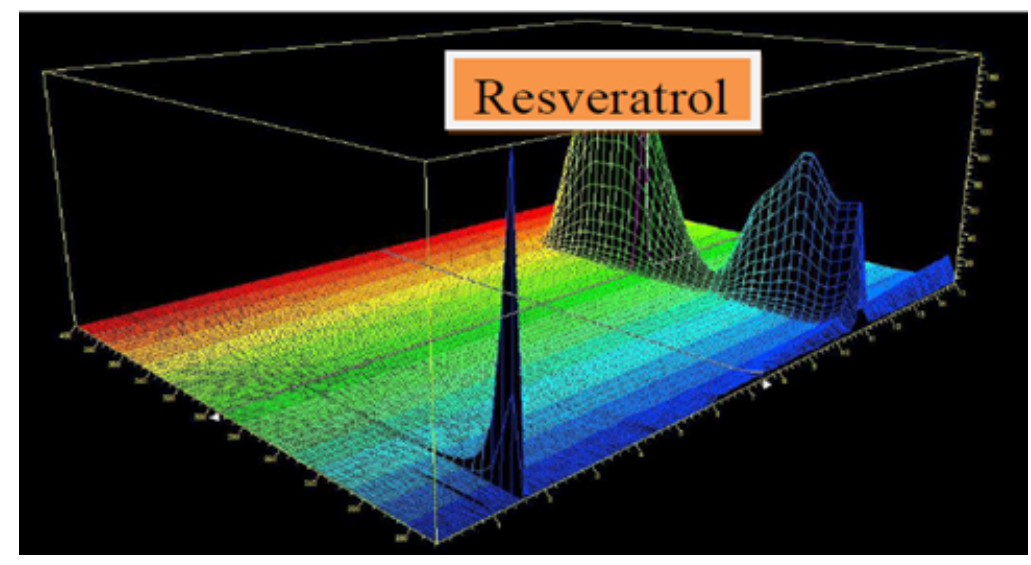

\begin{tabular}{|c|c|}
\hline Parameters & Obtained values \\
\hline Theoretical plates/column, halfwidth method & 5724 \\
\hline Symmetry & 1.068 \\
\hline USP tailing & 0.969 \\
\hline
\end{tabular}

Fig.3. 3D chromatogram of resveratrol

Table 1

SYSTEM SUITABILITY PARAMETERS OF RESVERATROL PEAK
The system suitability parameters of resveratrol peak are presented in Table 1.

Quantification was performed on the basis of the external standard method against average of 6 peak areas of standard solution chromatogram. The results are shown in figure 4.

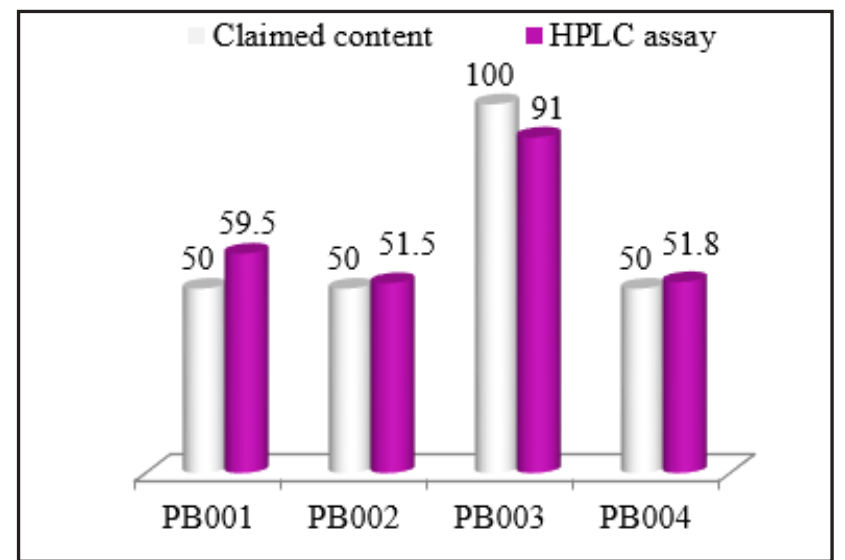

Fig.4. Resveratrol content of food supplements and vegetal extract

The content of resveratrol in the analyzed samples ranged between 91 and $119 \%$ from the claimed content on the productlabel. According to this data, samples PB001, PBO02 and PBO04 contained more resveratrol than was indicated on the label and the sample PBO03 contained less resveratrol than claimed. The precision of resveratrol was determined as the variation coefficient (RSD \%) for six successive injections of the standard solution which was 0.96 .

\section{Conclusions}

Resveratrol was successfully separated using the used and described method. The method provided a fast and cost efficient way for assaying resveratrol in food supplements.

Acknowledgement: The authors show their gratitude toSC Polisano Pharmaceuticals for providing support and research facilities for this work.

\section{References}

1.MALHOTRA, A., BATH, S., ELBARBRY, F.,Oxid Med Cel Longev, 2015,2015, p. 1.

2.OPREA, O. B., APOSTOL, L., BUNGAU, S., CIOCA, G., SAMUEL, A. D., BADEA, M., GACEU, L.,Rev. Chim. (Bucharest), 69, no. 1, 2018, p. 70. 3.FRUM, A., GEORGESCU, C., GLIGOR, F. G., LENGYEL, E., STEGARUS, D. I., DOBREA, C. M., TITA, O., Scientific Study \& Research Chemistry $\&$ Chemical Engineering, Biotechnology, Food Industry, 19, 1, 2018, p. 45.

4.KURĐVIETIENEA, L., STANEVIEIENEAB, I., MONGIRDIENEAC, A., BERNATONIENË, J., Medicina, 52, 3, 2016, p.148.

5.BOOCOCK, D. J., PATEL, K. R., FAUST, G. E.S, NORMOLLE, D. P., MARCZYLO, T. H., CROWELL, J. A., BRENNER, D. E., BOOTH, T. D., GESCHER, A., STEWARDA, W. P., J Chromatogr. B Analyt. Technol. Biomed Life Sci., 848, 2, 2007,p. 182.

6.FRANCIOSO, A., COSSI, R., FANELLI, S., MASTROMARINO, P., MOSCA, L., Int. J. Mol Sci., 18, 5, 2017, p. 967.

7.RICHARD, A., BAXTER, M. D., J. Cosmet. Dermatol., 7, 1, 2008, p. 2. 8.J UNCAN, A. M., HORGA, C. E. HODISAN, T., Studia UBB Chemia, 56 (LVI), 2, 2011, p. 195.

9.MUNTEAN, A. C., JUNCAN, A. M., MOISA, D. G., VONICA, A. L., RUS, L. L., MORGOVAN, C., GLIGOR, F. G. BUTUCA, A., STANILA, A., Mat. Plast., 56, no. 2, 2019, p. 360.

10.JUNCAN, A. M., Mat. Plast., 55, no. 4, 2018, p. 644.

11.DIAZ-GEREVINI, G. T., REPOSSI, G., DAIN, A., TARRES, M. C., DAS, U. N., EYNARD, A. R., Nutrition, 32, 2,2016, p. 174.

12.GHIBU, S., DECEA, N., MORGOVAN, C., MOGOSAN, C., FARMACIA, 61, 2, 2013, p. 420.

13.MORETON-LAMAS, E., LAGO-CRESPO, M., LAGE-YUSTY, M.A., LOPEZ-HERNANDEZ, J.Food Chem., 224, 2017, p. 219.

14.MORGOVAN, C., COSMA, S., BURTA, C., GHIBU, S., POLINICENCU, C., VASILESCU, D., Farmacia, 58, 4, 2010, p. 400.

15.ARDELEAN, F., VLASE, L., MOCAN, A. M., GHELDIU, A. M., ANTAL, D. S., TRANDAFIRESCU, C., MARGINEAN, O., DRAGAN, S., Rev. Chim. (Bucharest), 68, no. 2, 2017, p. 401.

16. BAXTER, R. A., J Cosmet Dermatol., 7, 1, 2008, p. 2.

17. SMOLIGA, J. M., BAUR, J. A., HAUSENBLAS, H. A., Mol. Nutr. Food, 55, 2011, p. 1129.

18. NEVES, A. R., LUCIO, M., LIMA, J. L. C., REIS, S., Curr Med Chem., 19, 11, 2012, p. 1663.

19. COTTART, C. L., NIVET-ANTOINE, V., BEAUDEUX, J. L., Mol. Nutr. Food Res., 58, 1, 2013, p. 7.

20. PANGENI, R., SAHNI, J. K., SHARMA, S., BABOOTA, S., Expert Opin. Drug. Deliv., 11, 8, 2014, p. 1285. 
21.MUÑOZ, O., MUÑOZ, R., BUSTAMANTE, S., BIOCHEM. PHARMACOL. (Los Angel)., 4, 5, 2015, p. 1.

22.CHRISTENSON, J., WHIBY, S. J , MELLOR, D., THOMAS, J., MCKUNE, A., ROACH, P. D., NAUMOVSKI, N., Metab. Syndr. Relat. Disord., 14, 7, 2016, p. 323.

23.RAUF, A., IMRAN, M., BUTT, M. S., NADEEM, M., PETERS, D. G., MUBARAK, M. S., Crit. Rev. Food Sci. Nutr., 58, 9, 2016, p. 1428.

24. RAUF, A., IMRAN, M., SULERIA, H. A. R., AHMAD, B., PETERS, D. G., MUBARAK, M. S., Food Funct., 8, 12, 2017, p. 4284.

25.PERRONE, D., FUGGETA ,M. P., ARDITO, F., COTTARELLI, A., DE FILIPPIS, A., RAVAGNAN, G., DE MARIA, S., LO MUZIO, L., Exp. Ther. Med., 14, 1, 2017, p. 3.

26.OZTURK, E., ARSLAN, A. K. K., YERER, M. B., BISHAYEE, A., Biomed. Pharmacother., 95, 2018, p. 230.

27. KOUSHKI, M., DASHATAN, N. A., MESHKANI, R., Clin. Ther., 40, 7, 2018, p. 1180.

28. GAMOH, K., NAKASHIMA, K., Rapid Commun. Mass Spectrom., 13, 1999, p. 1112.

29.SOUTO, A. A., CARNEIRO, M. C., SEFERIN, M., SENNA, M. J. H., CONZ, A., GOBBI, K., yJ. Food Compos. Anal., 14, 2001, p. 441.

30.GURBUZ, O., GOCMEN, D., DADDELEN, F., GURSO, M., AYDIN, S., SAHIN, I., BUYUKUYSAL, L., USTA, M., Food Chem., 100, 2007, p. 518. 31. CHEN, X., HE, H., WANG, G., YANG, B., REN, W., MA, L., YU, Q., Biomed. Chromatogr., 21, 2007, p. 257.

32. HUANG, H., ZHANG, J., CHEN, G., LU, Z., WANG, X., SHA, N., SHAO, B., LI, P., GUO, DA., Biomed. Chromatogr., 22, 2008, p. 421.

33. BIASUTTO, L., MAROTTA, E., GARBISA, S., ZORATTI, M., PARADISI, C., Molecules, 15, 2010, p. 6570.

34. GONCALVES, J., CAMARA, J. S., J. Sep. Sci., 34, 2011, p. 2376.

35. SOTO, M. E., BERNAL, J., MARTIN, M. T., HIGES, M., BERNAL, J. L., NOZAL, M. J., Food Anal. Methods, 5, 2012, p. 162.

36.HUANG, T. J., WENG, W. H., CHANG, T. L., CHUNG, J. P., J. Herbs Spices Med. Plants, 20, 4, 2014, p. 427.

37. LU, Q., ZHAO, Q., YU, Q. W., FENG, Y., J. Agric. Food Chem., 63, 19, 2015, p. 4771.

38.SHAO, Y., MARRIOTT, P., HUGEL, H., Chromatographia, 57, Suppl. 1, 2003, S349.

39. MONTES, R., GARCIA-LOPEZ, M., RODRIGUEZ, I., CELA, R., Anal. Chim. Acta, 673, 2010, p. 47.
40. CACHO, J. I., CAMPILLO, N., VINAS, P., HERNANDEZ-CORDOBA, M., J. Chromatogr. A, 1315, 2013, p. 21.

41.ARCE, L., TENA, M. T., RIOS, A., VALCARCEL, M., Anal. Chim. Acta, 359, 1998, p. 27.

42GU, X., CREASY, L., KESTER, A., ZEECE, M., J. Agric. Food Chem., 47, 1999, p. 3223.

43.DOBIASOVA, Z., PAZOUREK, J., HAVEL, J., Electrophoresis, 23, 2003, p. 263.

44.GALEANO DIZ, T., DURAN MERAS, I., AIRADO RODRIGUEZ, D., Anal. Bioanal. Chem., 387, 6, 2007, p. 1999.

45. BERNARDES, C. D., POPPI, R. J., SENA, M. M., Talanta, 82, 2010, p. 640.

46. MOLINA-GARCIA, L., RUIZ-MEDINA, A., FERNADEZ-DE CORDOVA, M. L., Anal. Chim. Acta, 689, 2011, p. 226.

47.ZHANG, H., XU, L., ZHENG, J., Talanta, 71, 2007, p. 19.

48. AIRADO-RODRIGUEZ, D., GALEANO-DIAZ, T., DURAN-MERAS, I., Food Chem., 122, 2010, p. 1320.

49.ZHANG, Q., QIAN, D., HE, H. B., AN, Y., WANG, T., Anal. Methods., 5, 2013, p. 3418.

50.GRANERO, A. M., FERNANDEZ, H., AGOSTINI, E., ZO, M. A., Electroanalysis, 20, 8, 2008, p. 858.

51.MING, L., PENG, T., TU, Y., Microchim. Acta, 183, 2016, p. 305.

52.XIANG, H., DAI, K., LUO, Q., DUAN, W., XIE, Y., Spectrochim. Acta A, 78, 1, 2011, p. 307.

53. MOISEI, A., TOTAN, M., GLIGOR, F. G., CRACIUN, I., TODORAN, N., CHIS A. A., POPA, D. E., Farmacia, 64, 4, 2016, p. 612.

54.HEGHES S. C., RUS, L. M., RUS, L. L., BOJITA, M. T., IUGA, C. A., Farmacia, 65, 5, 2017, p. 755.

55.MURESAN, M., BENEDEC, D., VLASE, L., OPREAN, R., TOIU, A., ONIGA, I., Studia UBB Chemia, 60 (LX), 1, p.127.

56.JUNCAN, A., M., HODISAN, T., Rev. Chim. (Bucharest), 62, no. 4, 2011, p. 415.

57. JUNCAN, A. M., RUS, L. L., GLIGOR, F. G., MORGOVAN, C., Rev. Chim. (Bucharest), 70, no. 2, 2019, p. 555.

Manuscript received: 21.06 .2018 